\title{
'SPOLIA' IN CONTESTO. IL RIUSO NELL’EPISCOPIO MEDIEVALE DI COMACCHIO
}

\section{SAURO GELICHI, RICCARDO BELCARI, DIEGO CALAON, ELENA GRANDI}

UDC: 726.7.033(450.456)

Preliminary communication

Manuscript received: 24. 01. 2011.

Revised manuscript accepted: 08. 03. 2011.

DOI: 10.1484/J.HAM.1.102270

S. Gelichi*

R. Belcari*

D. Calaon*

E. Grandi*

Italia

The archaeological excavations carried out from 2006 until 2008 in Piazza XX Settembre in Comacchio allow us to comprehend relative phases of the medieval Episcopal complex. Investigated contexts can be dated from the $6^{\text {th }} c$. to the Modern Age. The archaeological data concerning the first Bishop's church refer to the mid-eight century, proven by excavated cemetery area and porch. Original chapel was certainly placed under the modern Cathedral. Excavations brought to light several architectural elements and fragments of floor material, parts of the $8^{\text {th }}$ and $9^{\text {th }}$ century church, some reused in the later building. Different architectural elements of the first church (columns, capitals, marble slabs) were part of more ancient buildings, not necessarily of local origin. During the Carolingian period this early church was supplied with an altar screen. Between the end of the $10^{\text {th }}$ and the beginning of the $12^{\text {th }}$ century in front of the church new rectangular building was erected. By its shape, size and quality of used material, we can suggest that it corresponds to a part of the Romanesque Bishop's palace. Its walls were built with numerous spolia - parts of the earlier church. This demonstrates that the church itself has been completely reconstructed. The research of the architectural fragments and the data concerning some groups of architectural elements allows us to comprehend the quality of the early medieval church furniture. It is also possible to examine activities of re-use on a Romanesque construction site.

Key words: Comacchio, Episcopal complex, Middle Ages, reuse of stone material

\section{INTRODUZIONE}

Grazie alle ricerche archeologiche degli ultimi anni, Comacchio (fig. 2) si è rivelato essere un centro di straordinaria importanza per la conoscenza dell'economia dell'Italia e dell'Europa alto-medievale ${ }^{1}$. Lo studio di Comacchio, interpretabile come grande emporio dell'Adriatico settentrionale tra VIII e IX secolo (fig. 3), è stato possibile grazie anche ad un pluriennale progetto avviato dall'Università Ca' Foscari di Venezia e dalla Soprintendenza per i Beni Archeologici per l'Emilia Romagna, in stretta collaborazione con l'Amministrazione Comunale'2.

Un momento fondamentale di questo progetto è rappresentato dallo scavo condotto tra il 2006 e il 2008 in Piazza XX Settembre, ossia nel cuore dell'abitato dove oggi sorge la chiesa Cattedrale intitolata a San Cassiano (fig. 4). Qui, secondo un principio di persistenza topografica, si ipotizzava sorgesse anche la primitiva chiesa episcopale, ricordata dalle fonti scritte più attendibili a partire dal secolo VIII, non a caso proprio quando Comacchio si afferma definitivamente come emporio ${ }^{3}$.

L'indagine in quella che, a ragione, è stata battezzata come "Isola del vescovo", ha restituito un'interessante sequenza stratigrafica di lungo periodo. Lo scavo ha permesso di fare chiarezza sul momento della nascita e del successivo sviluppo di un abitato nato all'interno di una laguna, poco lontano dalla fascia litoranea, su alcuni dossi sabbiosi di recentissima formazione.

I contesti e i materiali che si prenderanno in esame in questa occasione provengono appunto da questo scavo.

\section{LA SEQUENZA INSEDIATIVA DAL VI SECOLO AL X SECOLO}

Le prime tracce di un'occupazione stabile di questo sito si datano tra la fine del VI e gli inizi del VII secolo e sono rappresentate da un edificio in legno che aveva funzioni

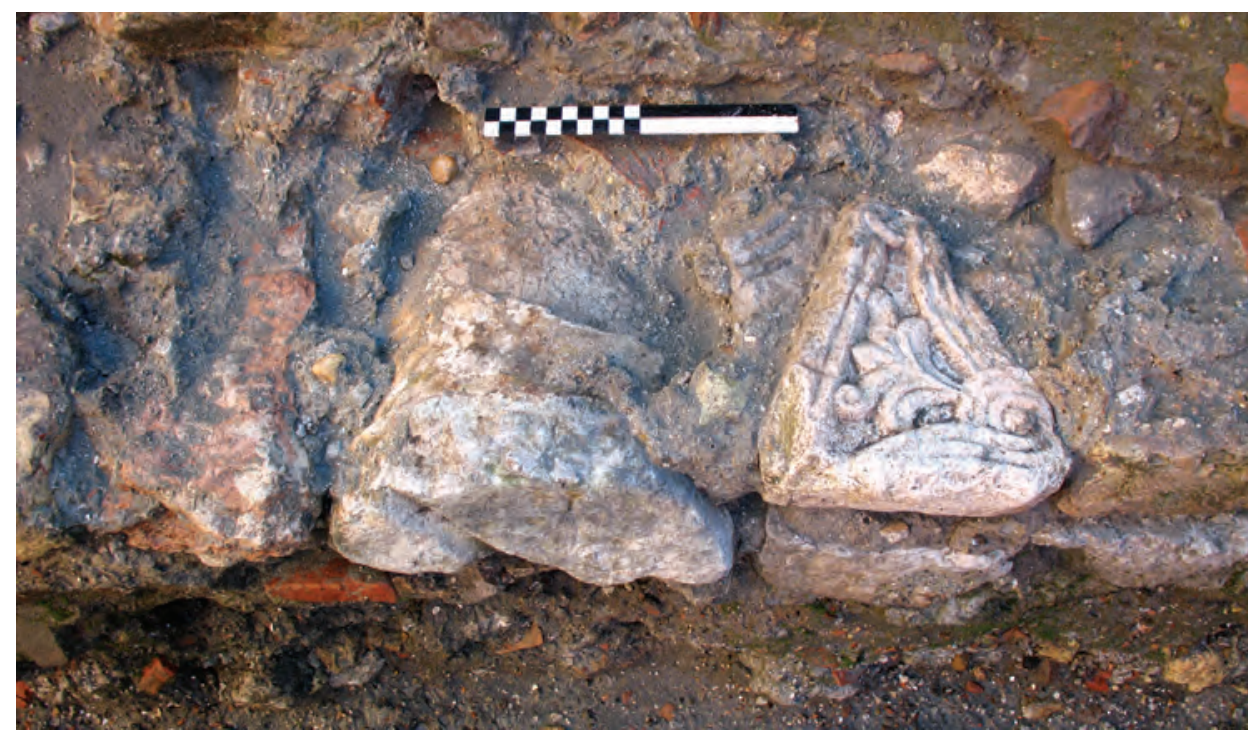

Fig. 1. Spolia in opera nelle murature del Palazzo Vescovile rinvenute negli scavi urbani di Comacchio (Ferrara - Italia) 


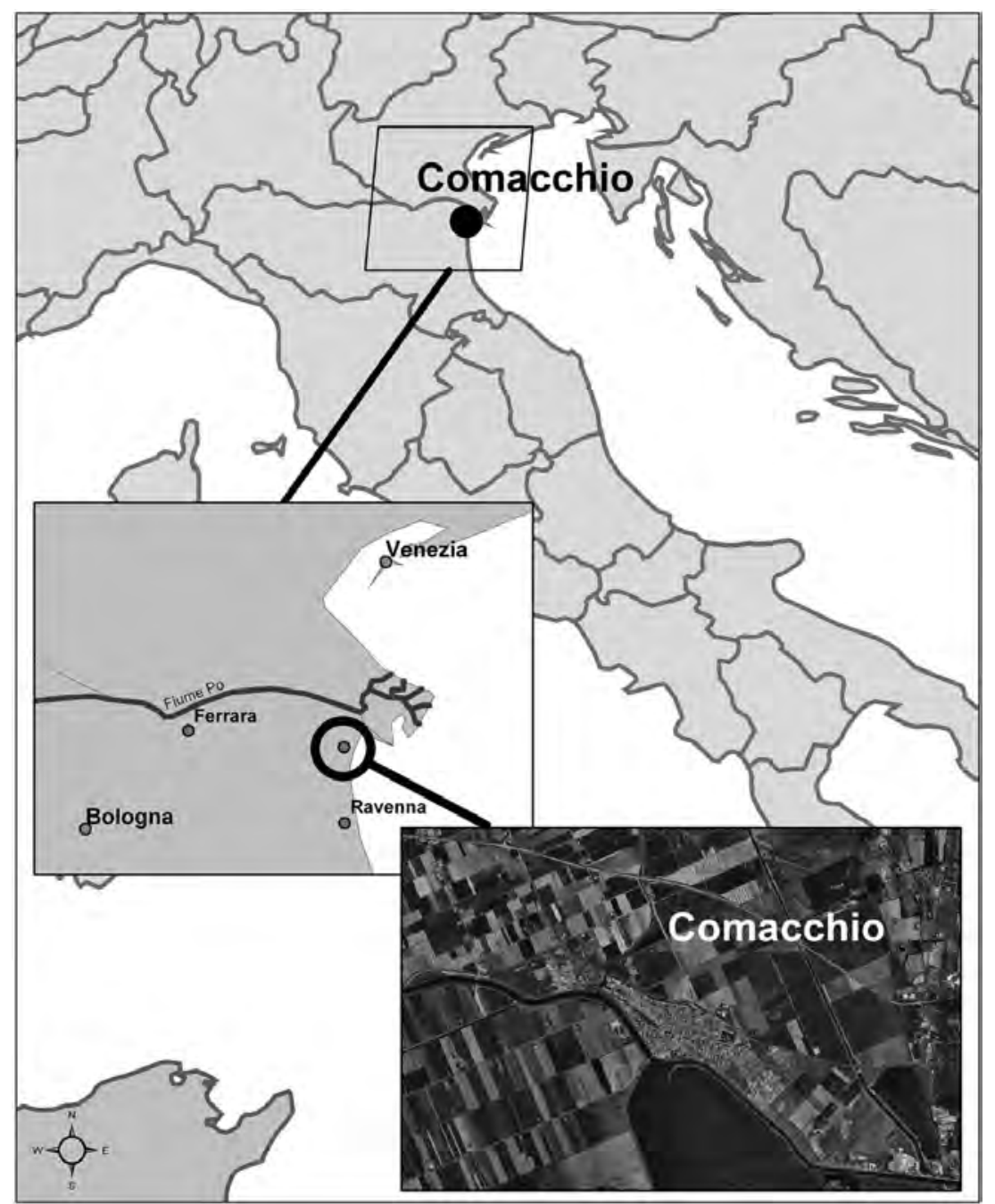

Fig. 2. Localizzazione di Comacchio (Ferrara - Italia)

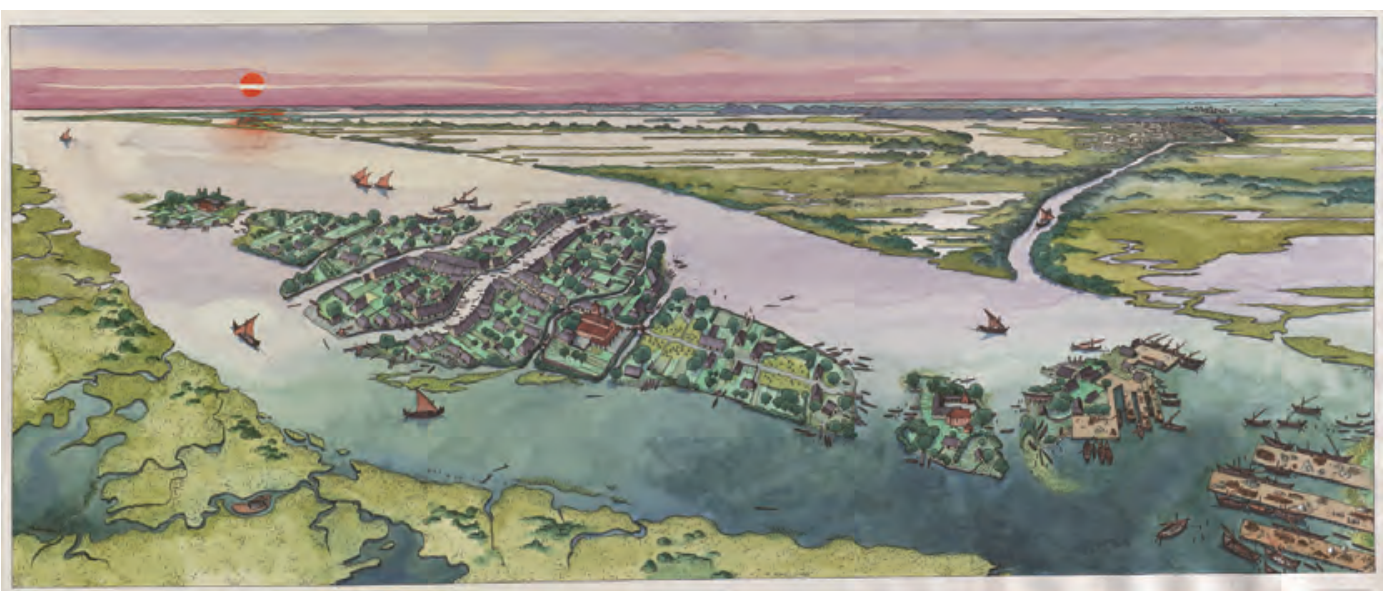

Fig. 3. Comacchio: l'abitato e il porto nell'alto medioevo. Disegno di R. Merlo

abitative. Nel corso del VII secolo questa struttura venne obliterata e tutta quanta l'area trasformata per accogliere un impianto produttivo nel quale si lavorava il ferro e il vetro. La produzione del vetro (testimoniata da scorie, gocciolature e colletti di soffiatura, cioè semilavorati) era finalizzata alla realizzazione di manufatti di uso corrente, come recipienti a soffiatura, tra cui calici, ma anche di oggetti di qualità superiore, come cammei in pasta di vetro (fig. 5), la cui presenza è certificata dal rinvenimento di una matrice ${ }^{5}$.

Successivamente, a partire dal secolo VIII, si assiste ad un'ulteriore radicale trasformazione d'uso dell'area che da artigianale diviene cimiteriale. Il cimitero, attivo fino alla prima metà del IX secolo, costituisce il primo evidente segno dell'esistenza, nelle vicinanze, di un edificio di culto, nel quale non abbiamo difficoltà a riconoscere la prima chiesa episcopale (fig. 6). Tuttavia lo scavo non ha consentito di individuare nessuna traccia di questa chiesa che, verosimilmente, doveva trovarsi nell'area dove ancora oggi è il Duomo di Comacchio.

Le successive evidenze archeologiche, riconducibili alla seconda metà del IX secolo, si riferiscono all'obliterazione della necropoli mediante la stesura di ampi riporti di terreno e alla creazione di un terrapieno sormontato da una palizzata. Si provvide, dunque, a delimitare il margine 


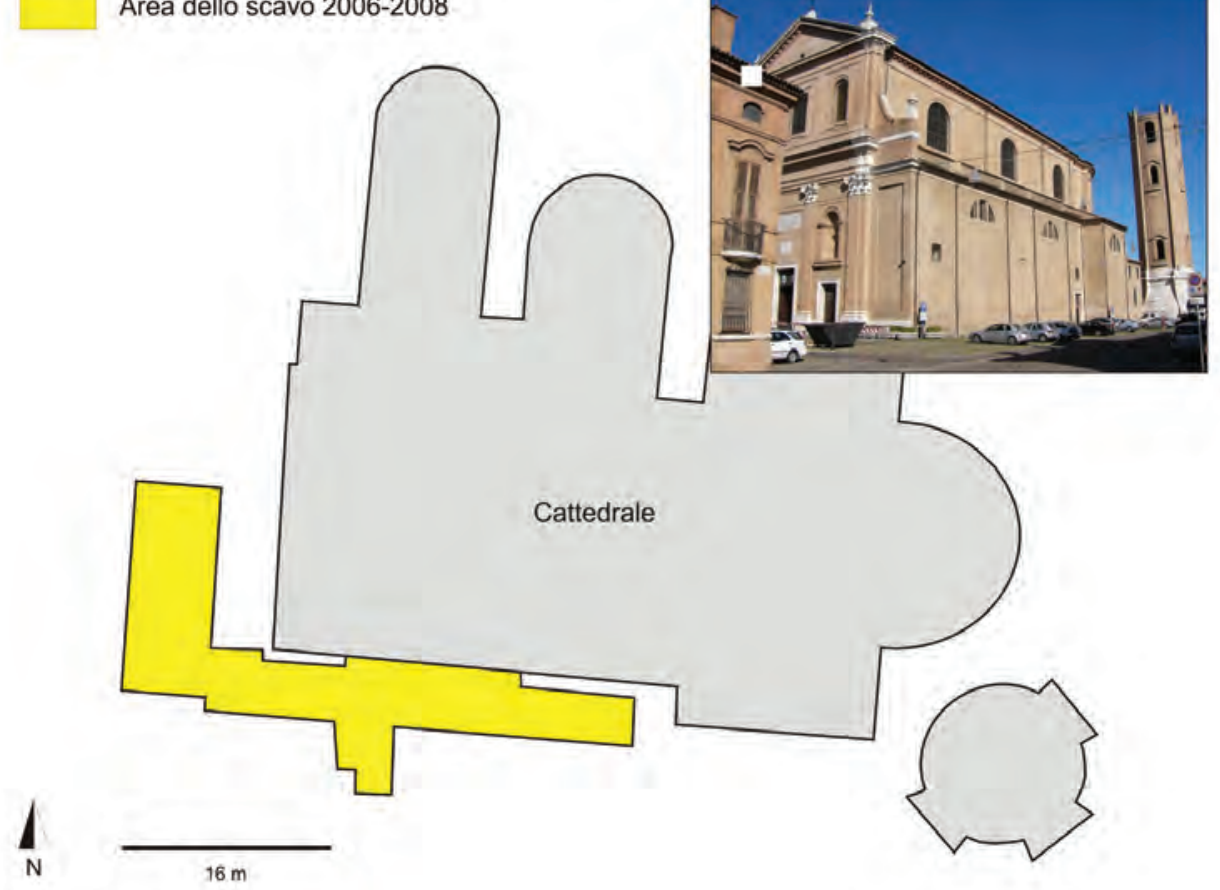

Fig. 4. Localizzazione dello scavo di P.zza XX Settembre; la Cattedrale di San Cassiano
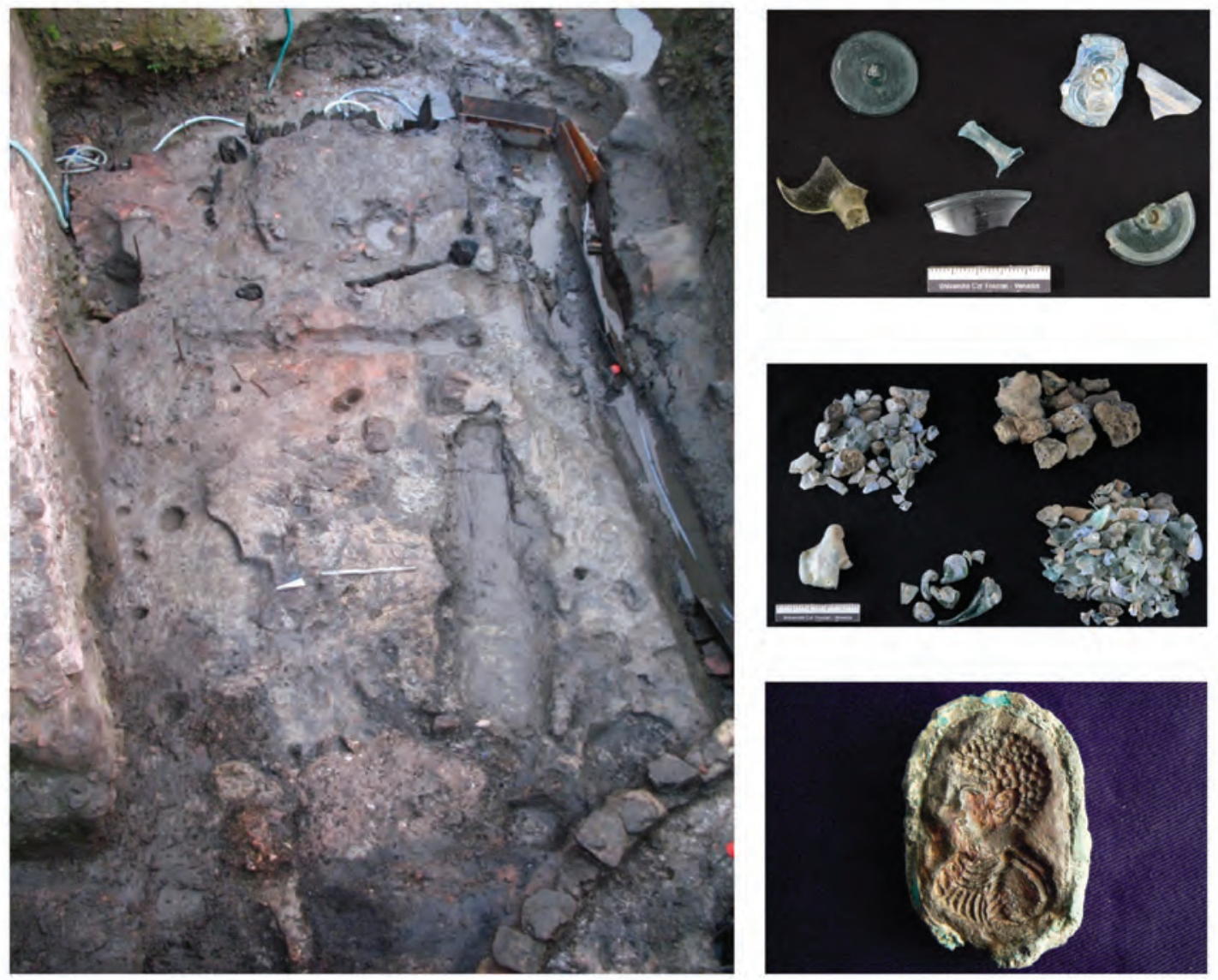

Fig. 5. Parte delle strutture pertinenti all'impianto produttivo; frammenti di calici altomedievali; gocce di fusione e scorie di lavorazione del vetro; matrice per cammeo

occidentale dell'isola, circoscrivendo uno spazio difeso e recintato da un canale.

In un momento databile tra la fine del X e l'inizio dell'XI secolo, quest'area fu ancora interessata da ulteriori radicali interventi edilizi. Si realizzó, cioè, un ampio edificio in mu- ratura, a pianta rettangolare, che doveva svilupparsi su due piani. Questo edificio (che lo scavo ha messo in evidenza per una buona parte) è stato interpretato come una porzione di un palazzo episcopale. Tale complesso era adiacente alla chiesa, orientato ad "ala" e probabilmente si affacciava 


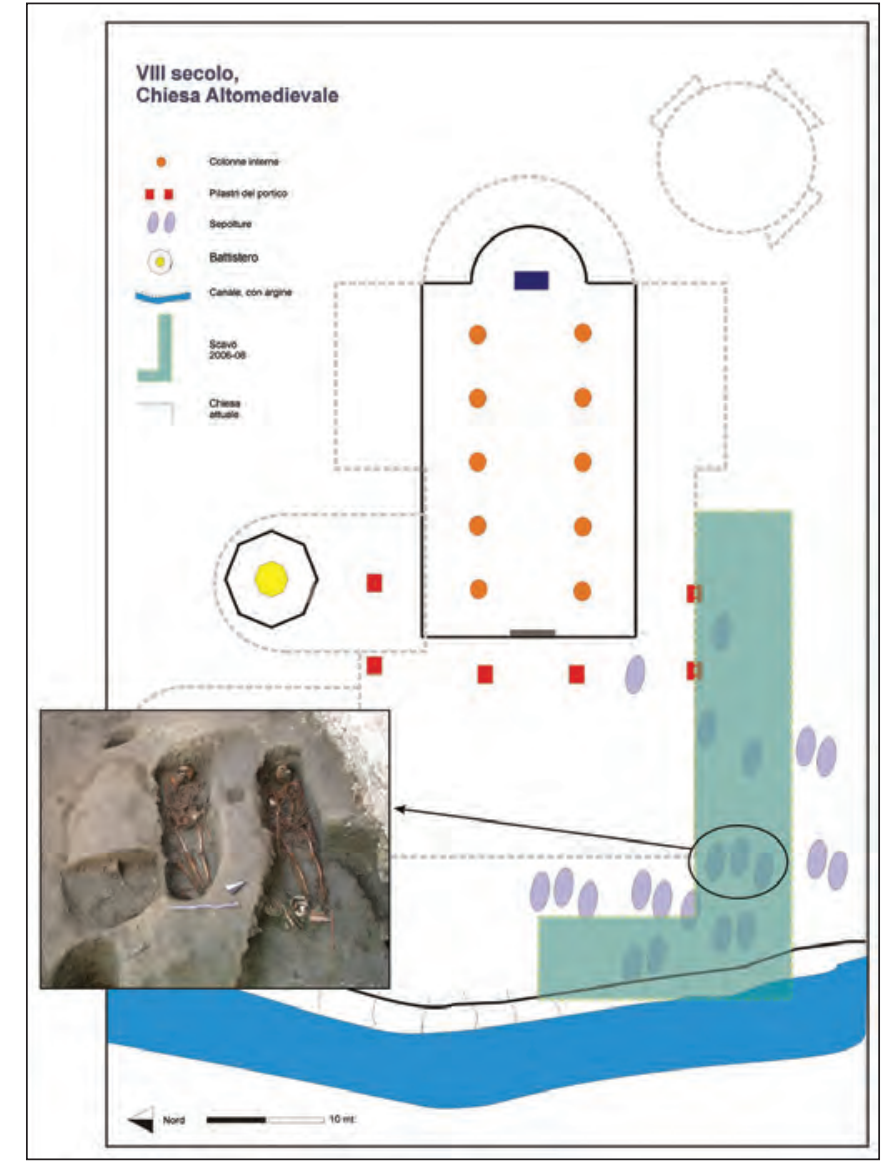

Fig. 6. Planimetria ipotetica della chiesa vescovile altomedievale e sepolture in nuda terra

con un portico sul sagrato stesso della cattedrale (fig. 7). Non è da escludere che esistesse una seconda ala sul lato opposto rispetto all'entrata della chiesa. La piazza aveva come punto d'accesso un ampio canale navigabile, che si trovava di fronte all'entrata della chiesa.

Le fasi successive della sequenza interessano i periodi di vita del palazzo episcopale e poi la sua distruzione, avvenuta per far posto, ancora una volta, ad uno spazio di natura funeraria: un cimitero che, questa volta, giunge fino alle soglie dell'età moderna.

In questo contributo ci soffermeremo sulle fasi databili tra VIII e XI secolo, che corrispondono al periodo in cui furono in opera gli spolia di cui vorremmo occuparci.

\section{LA CHIESA ALTOMEDIEVALE DI COMACCHIO: LE INFORMAZIONI ARCHEOLOGICHE}

Gli scavi non hanno permesso di acquisire informazioni dirette sulla pianta della primitiva chiesa episcopale che, come abbiamo detto, supponiamo si trovi al di sotto del poderoso Duomo attuale, eretto verso la fine del XVII secolo. Per quanto non esistano strutture in alzato che possano aiutarci a determinare forma e dimensioni della chiesa altomedievale, esistono chiari indizi che ne certificano la presenza, che ci aiutano a stabilirne l'orientamento e a proporre alcune congetture sul suo arredo: il cimitero di tardo VIII secolo, gli spolia (rinvenuti principalmente reimpiegati negli edifici di età romanica e in alcuni livelli di riporto databili al $\mathrm{X}$ secolo), le numerose attestazioni relative ai pavimenti in mosaico e in opus sectile ${ }^{6}$. Non possiamo dimenticare, infine, l'abbondante documentazione di epoca tardo medievale e moderna, come le descrizioni contenute nelle visite pastorali e la cartografia, che qui tuttavia dob-

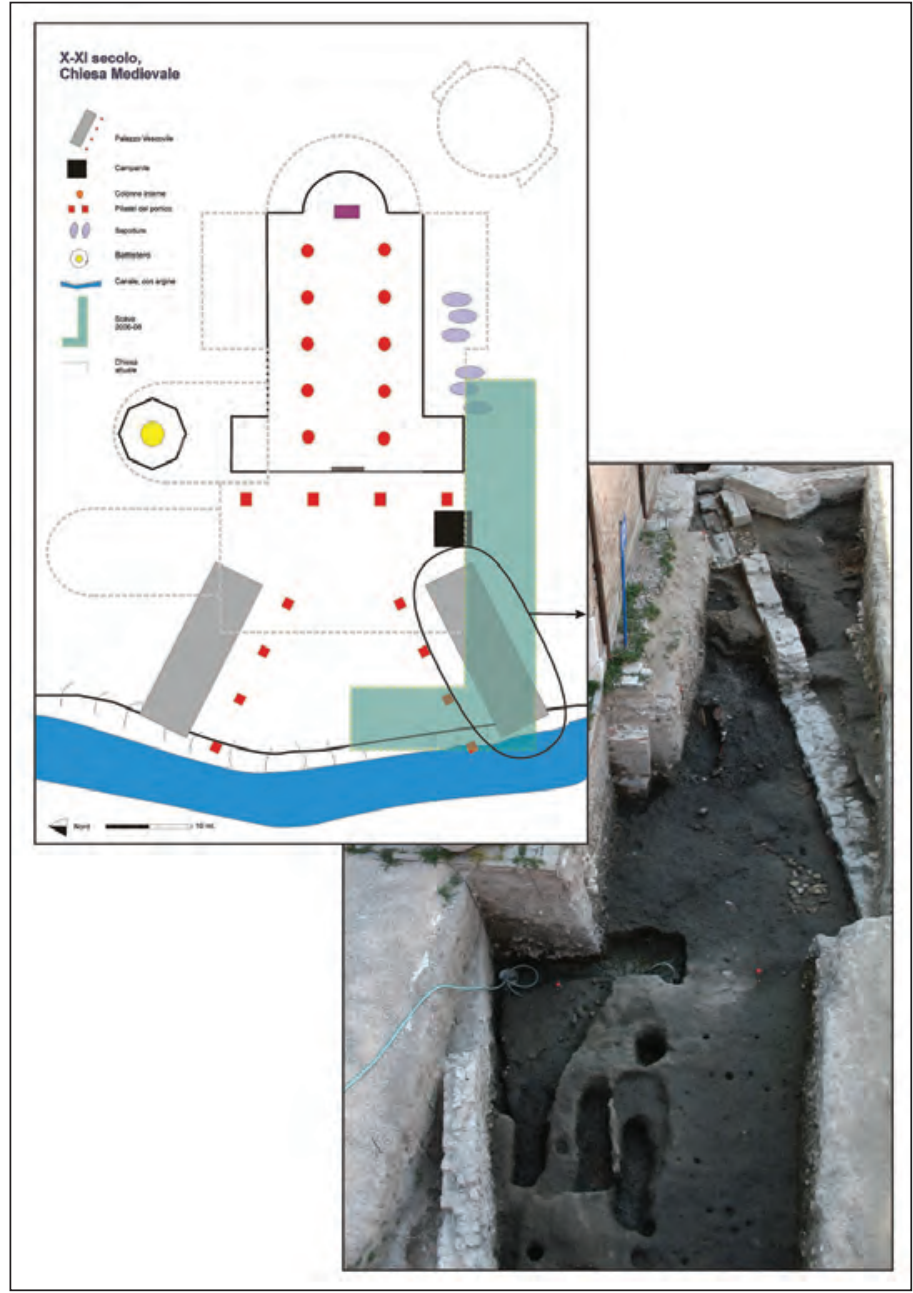

Fig. 7. Planimetria ipotetica del complesso episcopale (XI secolo); i perimetrali del palazzo vescovile

biamo tralasciare, ma che ci permettono almeno di asserire con sicurezza che l'abside della chiesa altomedievale doveva all'incirca coincidere con quella del Duomo odierno.

Di notevole interesse sono gli spolia rinvenuti nella fasi di tardo X e inizio XI secolo. Si tratta soprattutto di resti di colonne, colonnine, capitelli, frammenti di trabeazioni, lastre e pilastrini, per un totale di circa 150 frammenti. Molto numerose sono anche le tessere musive (diverse centinaia) e le lastrine marmoree (fig. 8). Proprio questi elementi ci informano non solo dell'esistenza della chiesa, ma ci permettono anche di formulare qualche riflessione sulle sue componenti strutturali e su quelle decorative.

Innavanzitutto, è interessante osservare come un certo numero di materiali architettonici (capitelli, rocchi e frammenti di colonna, lastre marmoree) - cioè circa il 10-15\% del totale - e due frammenti pertinenti a un arredo liturgico, siano attribuibili ad un orizzonte cronologico più antico rispetto a quello a cui assegniamo la chiesa (VIII secolo). Si tratta, infatti, di oggetti riferibili alla tarda antichità che, dunque, dobbiamo supporre già impiegati come elementi di spoglio nell'originaria chiesa episcopale e provenienti da edifici più antichi ubicati nel territorio o arrivati direttamente da Ravenna.

L'ipotesi che questi pezzi siano stati prelevati da un altro luogo (che non la chiesa cattedrale) per essere usati nelle murature dell'episcopio durante i rifacimenti di epoca romanica ci pare poco plausibile, per tre principali motivi.

In primo luogo per il fatto che tutti questi pezzi sono stati ritrovati in un deposito composto da macerie (fra cui 

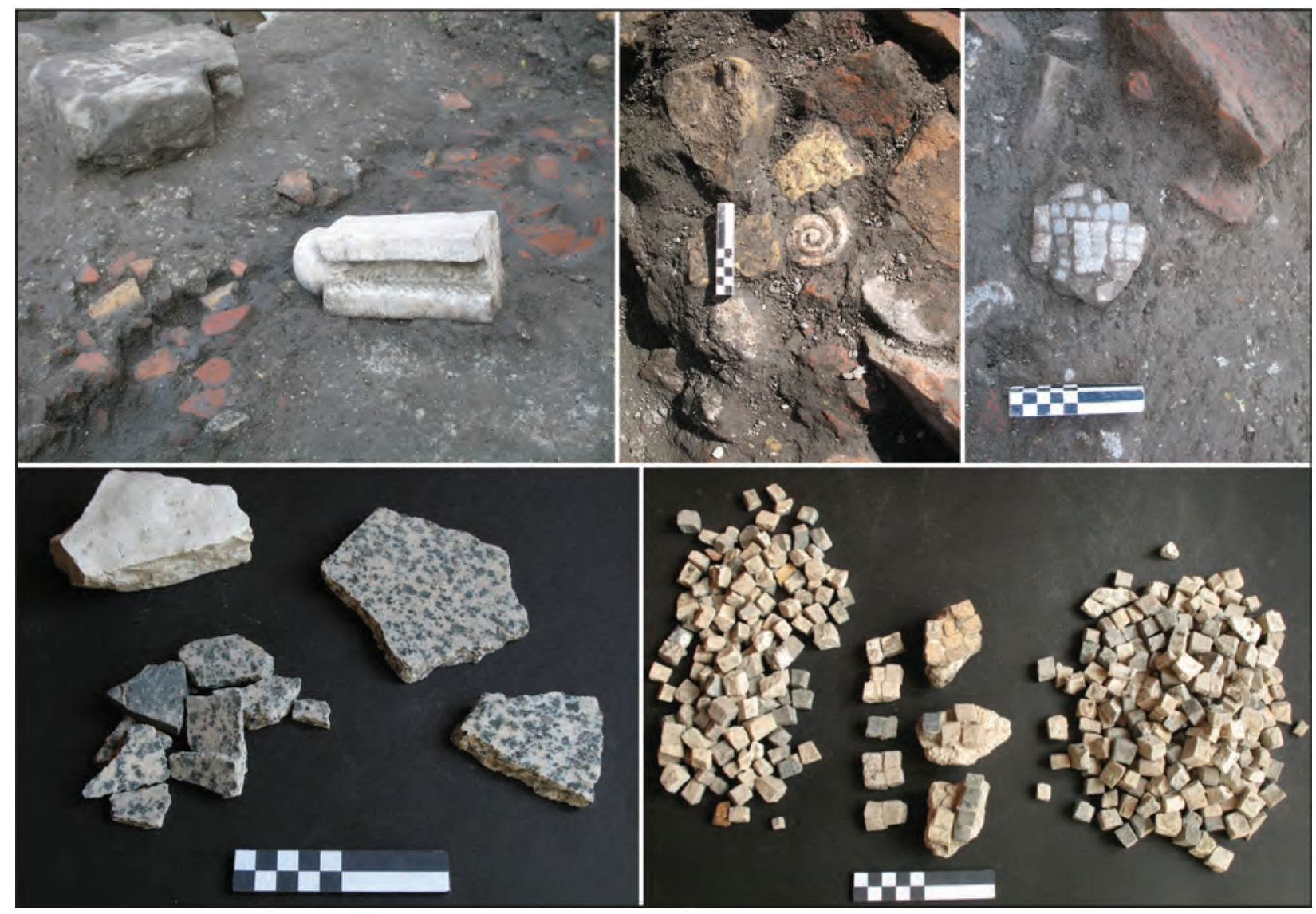

Fig. 8. Frammento di pilastrino rinvenuto in scavo; frammento di elemento scultoreo; porzione di pavimento musivo; lastrine marmoree e tessere musive pavimentali in marmo bianco e grigio

anche pezzame laterizio) databile alla fine $\mathrm{X}$ secolo e riferibile al momento in cui la vecchia chiesa alto-medievale fu, almeno parzialmente, ricostruita. Gli spolia, sia gli elementi databili ai secoli VIII e IX, che quelli più antichi, vennero tutti impiegati nelle fondazioni delle strutture del nuovo impianto vescovile e utilizzati in maniera indifferenziata. Pare dunque molto verosimile che i costruttori della nuova cattedrale abbiano fatto un uso indistinto di macerie prodotte dalle demolizioni e disponibili in loco.

Secondariamente, le murature della chiesa altomedievale si componevano principalmente (se non esclusivamente) di materiale di reimpiego, utilizzato quasi sempre a livello frammentario: in particolare tegole con spallette e mattoni di modulo romano, che sono stati rinvenuti in grande quantità in scavo. Sembra dunque ipotizzabile una grande attività di spoglio al momento della costruzione della primitiva chiesa durante il secolo VIII, peraltro secondo una prassi molto diffusa in quel periodo.

Dai frammenti riconducibili a fusti di colonna, è possibile ipotizzare che questi elementi architettonici fossero di grandi dimensioni e riconoscerne in via preliminare i marmi di importazione con cui furono realizzati, probabilmente greci e orientali, tra i quali cipollino, verde antico, pavonazzetto $^{7}$. Altri frammenti di piccole dimensioni recuperati dovevano verosimilmente appartenere a rivestimenti parietali o pavimentali, ossia a sectilia e crustae (fig. 9.c-d), realizzati in materiali pregiati come il giallo antico, il verde antico, il rosso e altri marmi di importazione ${ }^{8}$. Questi manufatti trovano confronti con materiali riferibili alla tarda antichità, documentati a Ravenna o, ancora in ambito regionale, a Reggio Emilia ${ }^{9}$.

Da segnalare, infine, la presenza di un capitello databile tra la fine del V e i primi anni del VI secolo, decorato a giorno con foglie mosse dal vento del tipo "a farfalla" e rilavorato per essere destinato ad altro uso e, come tale, già di reimpiego (fig. 10). L'elemento, del quale sono state recuperate due foglie e un frammento dell'abaco con motivo ad ovoli e frecce, trova puntuali analogie con capitelli di età teodoriciana originariamente in opera in edifici ravennati e ora al Museo Nazionale di Ravenna ${ }^{10}$, o nel palazzo in piazza del Popolo costruito dai Veneziani nel $1461^{11}$, ma anche, con qualche differenza, con quelli pertinenti al colonnato di S. Apollinare in Classe ${ }^{12}$.

Sulla scorta di questi dati possiamo supporre che l'edificio vescovile fosse costruito con mattoni di reimpiego (che potevano provenire anche da monumenti romani in abbandono nel territorio) e con materiale di spoglio più pregiato, come marmi (colonne e capitelli), che non è improbabile fossero arrivati direttamente da Ravenna.

\section{L'ARREDO SCULTOREO DI IX SECOLO}

Nel IX secolo gli indicatori materiali che provengono globalmente dal territorio di Comacchio e non solo dallo scavo, ci descrivono complessivamente un centro fiorente, ancora assai attivo sul piano commerciale e ben collegato sia ai mercati mediterranei che a quelli padani. Anche sul piano topografico, Comacchio sembra essere oramai una realtà ben definita: un centro lagunare con due grandi poli d'attrazione, uno rappresentato dalle infrastrutture portuali ad Occidente e l'altro dall'isola vescovile, con la chiesa episcopale, verso Oriente.

Il programma decorativo della cattedrale, proprio nel IX secolo, si dotò di una recinzione presbiteriale; sono infatti da ricondurre ad essa i frammenti recuperati pertinenti a lastre, pilastrini, cornici e capitelli, nonché a una trabeazione 

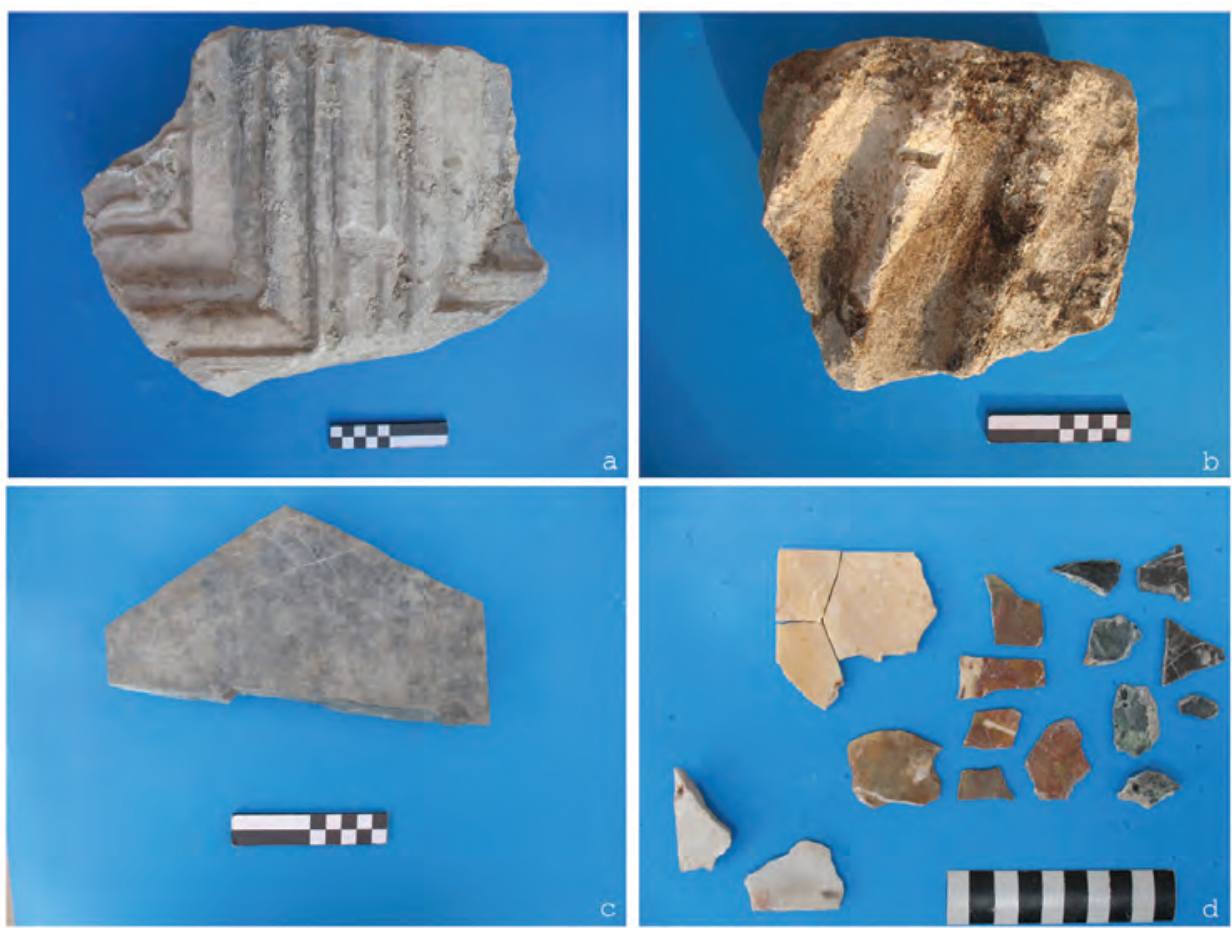

Fig. 9. Spolia tardo antichi rinvenuti nel contesto indagato: a) frg. lastra (elemento di arredo ?); b) frg. sarcofago strigilato; c) frg. formella esagonale di sectilia pavimentale; d) frgg. crustae marmoree di rivestimento (foto R. Belcari)
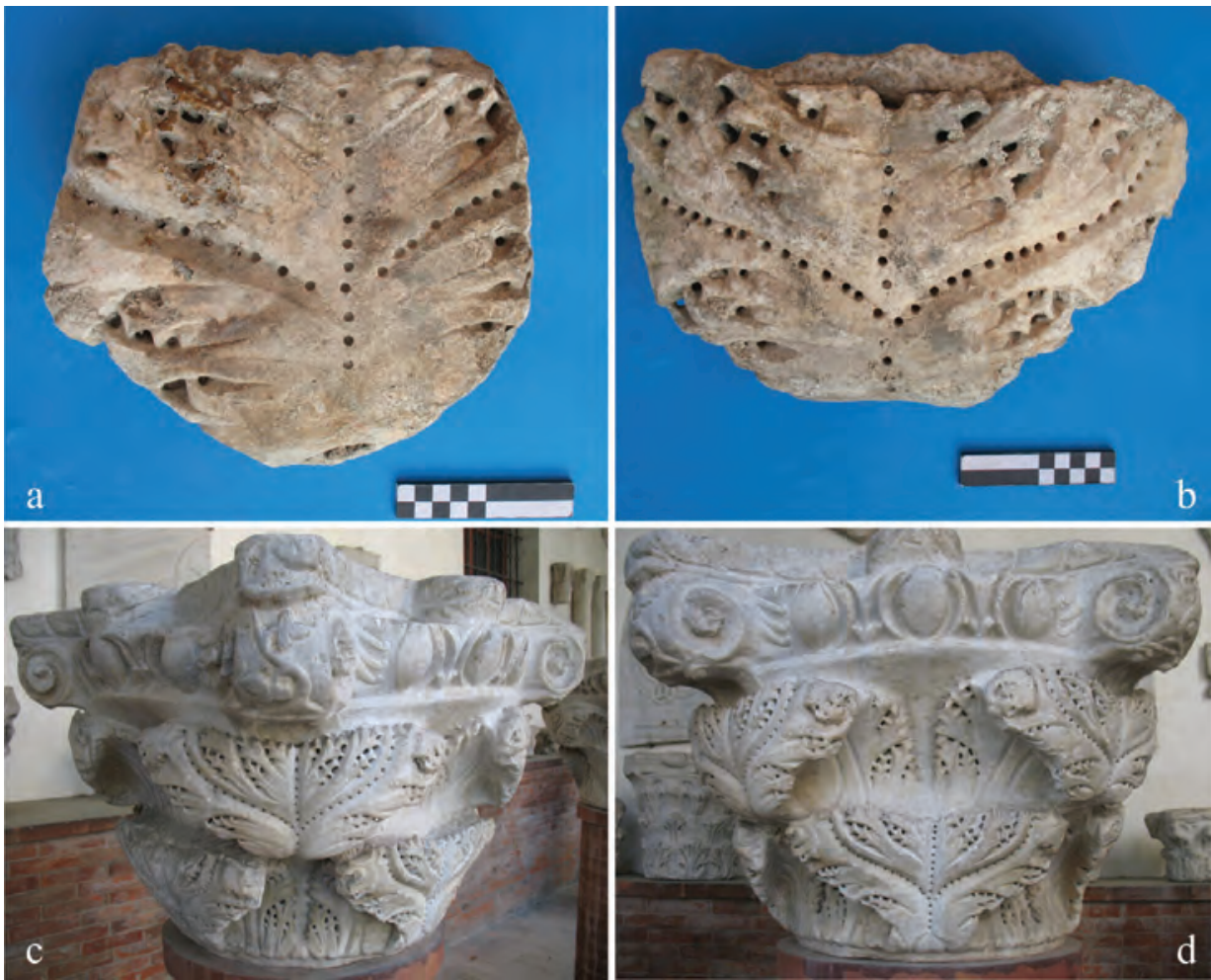

Fig. 10. a-b) Foglie con decorazione a giorno pertinenti capitello, recuperate durante le indagini archeologiche; $c$-d) Capitello con foglie mosse dal vento del tipo "a farfalla", inizio VI secolo, Museo Archeologico Nazionale, Ravenna (foto R. Belcari)

dove si conservano alcune lettere di un'iscrizione dedicatoria $^{13}$. Gli elementi recuperati, interessati da decorazione a intreccio, corrispondono a una quarantina di frammenti, di media o modesta dimensione (figg. 11-12). L'acquisizione, che crediamo interessante in primo luogo proprio per il contesto di rinvenimento, arricchisce, anche in termini quantitativi, il panorama della produzione scultorea coeva, e fornisce dati relativi ai litotipi, agli strumenti e alle tecniche impiegate. Gli artefici ricorsero a diversi materiali lapidei, al momento individuabili come pietra d'Istria, marmo bianco microcris- tallino di presumibile reimpiego e altri due diversi litotipi, uno dei quali classificabile genericamente come calcare. La recensione analitica delle tracce di lavorazione visibili sulle superfici consente di documentare l'impiego di strumenti a punta, a percussione probabilmente indiretta, utilizzati nella preliminare definizione dei pezzi, nonché di lame, piane e dentate. Sono inoltre presenti alloggi ricavati negli elementi destinati allinserimento di staffe e perni metallici per l'assemblaggio della struttura. Di un certo interesse risulta il confronto con i materiali del territorio preceden- 

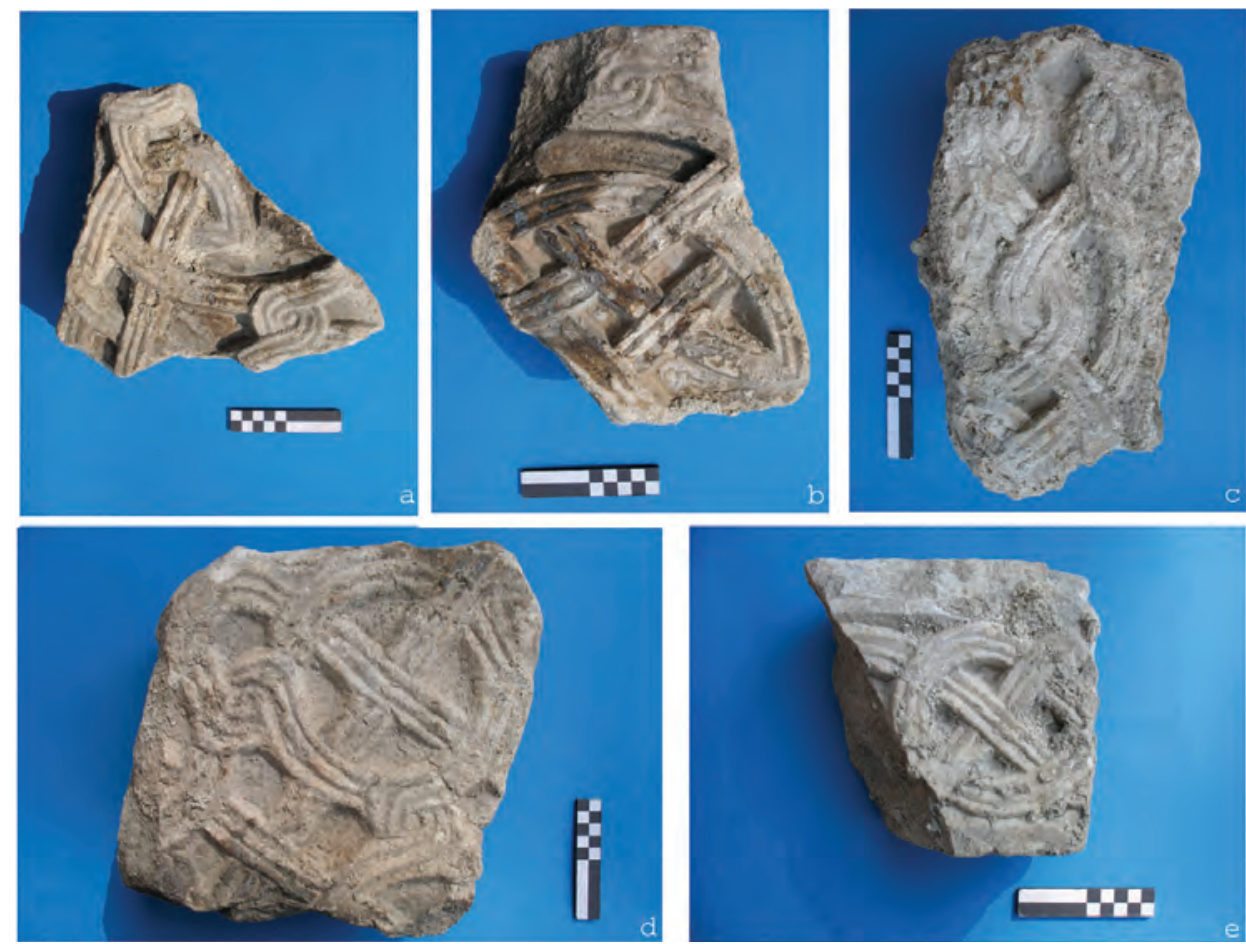

Fig. 11. Elementi frammentari di arredo liturgico con decorazione a intreccio: a-b-d) frgg. lastre-plutei; c) frg. pilastrino con decorazione su due lati; e) frg. cornice (foto R. Belcari)
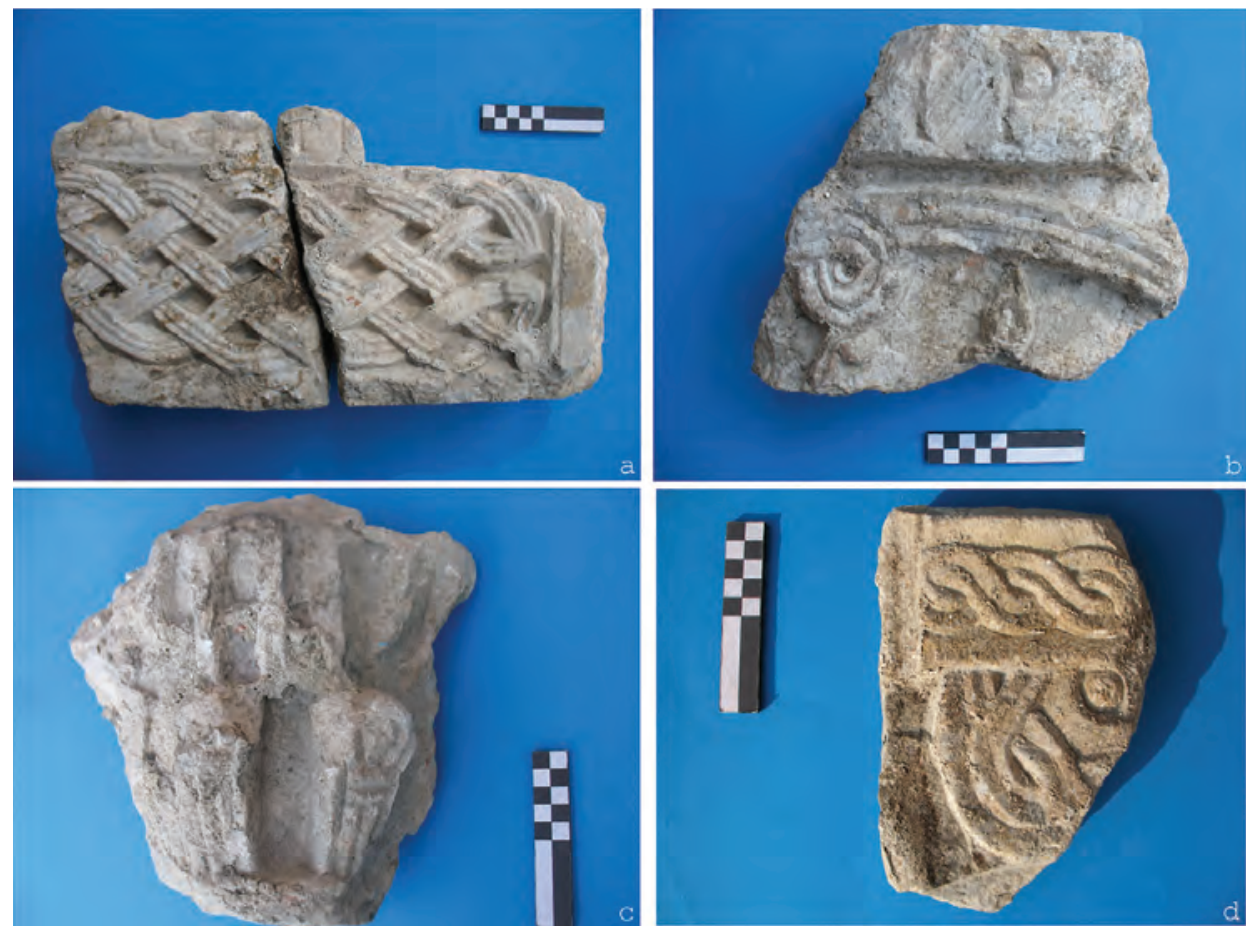

Fig. 12. Elementi frammentari di arredo liturgico: ab) frgg. trabeazione e lastra con decorazione a intreccio e iscrizioni mutile; c) frg. capitello con corona di archetti; d) frg. lastra/pluteo con tenone (foto R. Belcari)

temente noti ${ }^{14}$. Ad esclusione di alcuni confronti generici, limitati ai motivi decorativi ${ }^{15}$, gli elementi non sembrano infatti presentare analogie evidenti ${ }^{16}$. Risultando consistere in motivi geometrici di superficie, realizzati con nastri viminei bisolcati ad intreccio, in varie soluzioni e con differente resa, nonché in elementi fitomorfi stilizzati a questi combinati, trovano altresì generici confronti con la produzione scultorea connessa agli elementi di arredo liturgico coeva, talora più puntualmente riscontrabili nell'area alto adriatica ${ }^{17}$.

Nel complesso, la qualità dei frammenti riconducibili ad elementi architettonici e di arredo liturgico, unitamente alla presenza di tessere pavimentali bianche e nere, lastrine marmoree, nonché tessere vitree parietali, suggeriscono un apparato decorativo consono a una committenza in grado di destinare risorse economiche in imprese edilizie evidentemente rappresentative ${ }^{18}$.

\section{L'ETÀ ROMANICA}

Come abbiamo detto, all'età romanica si data la costruzione di un ampio corpo di fabbrica in muratura che verosimilmente costituiva una parte del palazzo episcopale.

L'edificio, a pianta rettangolare, ampio circa 70 metri quadrati, era orientato est-ovest e presentava, sul lato 


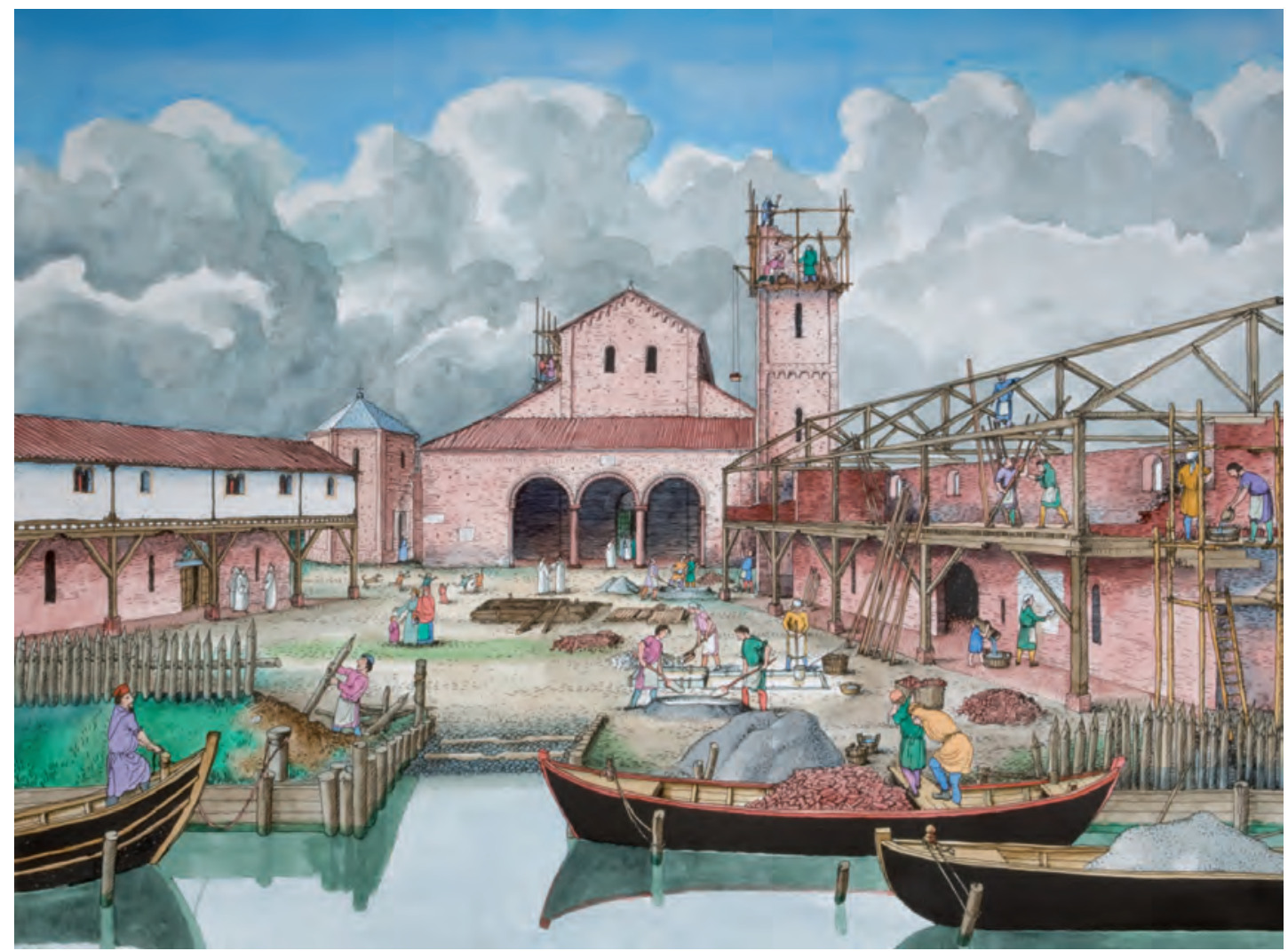

Fig. 13. L'episcopio di età romanica (XI secolo). Disegno di R. Merlo

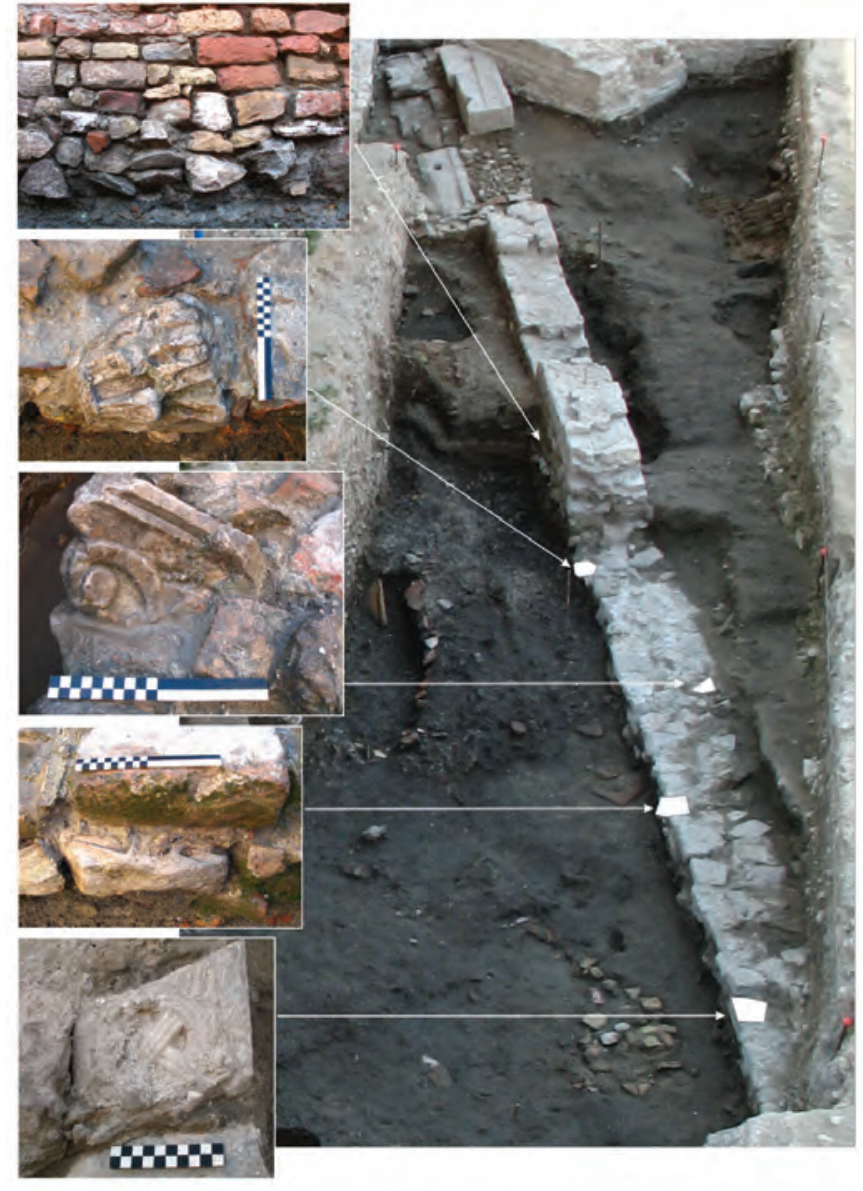

Fig. 14. Perimetrale meridionale del palazzo vescovile; particolari dei materiali costruttivi di reimpiego

interno prospiciente il sagrato, un portico con pilastri in muratura (fig. 13). Le dimensioni planimetriche, gli elementi strutturali e la tipologia delle fondazioni consentono di ipotizzare che fosse un edificio a due piani. Il piano terra, di cui non si sono conservate le pavimentazioni e privo di segni evidenti di attività, potrebbe essere stato destinato a magazzino. Il piano superiore, invece, doveva essere destinato ad uso residenziale.

Lo scavo ha riportato alla luce ampie porzioni dei perimetrali di questo edificio, parzialmente conservati in elevato. Così è possibile farsi un'idea delle tecniche costruttive. L'alzato era caratterizzato da corsi non troppo regolari di mattoni di modulo romano, frammentati e di spessori molto variabili, uniti a rari frammenti di tegole con alette e legati da malta friabile. Le fondazioni, invece, utilizzavano sempre spezzoni di mattoni associati, questa volta, a molti frammenti lapidei. La qualità, le forme e le decorazioni delle pietre e dei marmi riutilizzati ci fa supporre che la componente maggioritaria fosse composta da elementi architettonici, già danneggiati, provenienti dalla chiesa distrutta o pesantemente lesa.

I materiali di dimensioni più grandi, soprattutto resti di colonne, erano collocati nella parte basale della muratura, in modo da rendere più stabile l'edificio costruito sopra depositi limo-sabbiosi non compatti. La fondazione, che appare più larga dell'alzato, era poi caratterizzata da un profilo accentuatamente irregolare, per il fatto che questi elementi erano stati impiegati senza essere risagomati, mentre quelli di dimensioni più contenute (frammenti di lastre e di capitelli, etc...) erano stati gettati senza alcuna regolarità e frammisti a pezzame di laterizio con abbondante malta (fig. 14).

Tutti i pezzi lapidei reimpiegati, anche quelli decorati, dunque, non erano visibili nell'alzato (e anche nelle fondazioni avevano spesso la parte decorata verso l'interno): è dunque indiscutibile un loro utilizzo come semplice materiale da costruzione.

I soli spolia che forse erano visibili nelle murature di età romanica erano murati nell'unica porzione di chiesa ripor- 


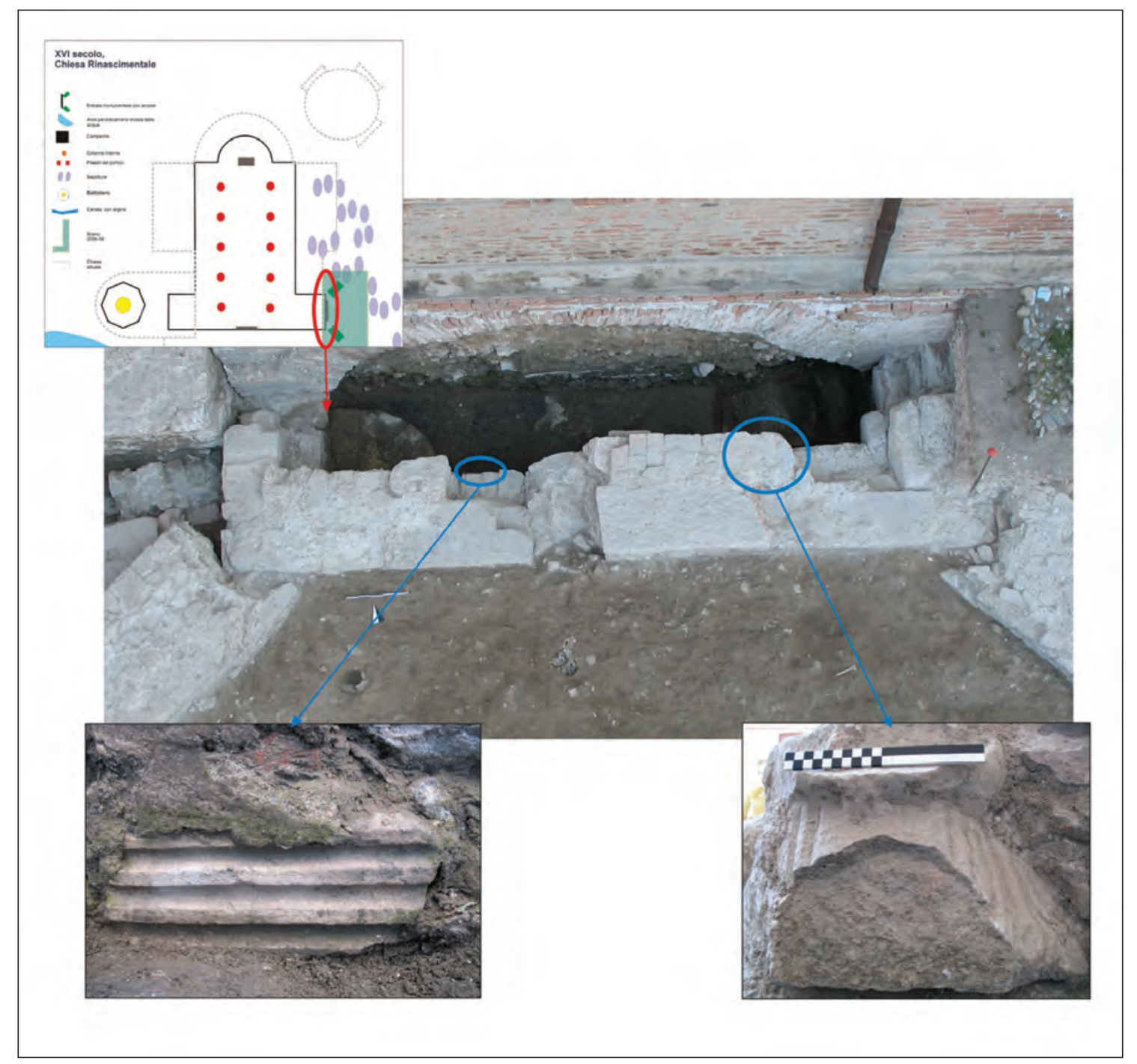

Fig. 15. Elementi reimpiegati nella murature della chiesa di età romanica e nei rifacimenti di età moderna

tata alla luce nello scavo, cioè una parte del nartece non obliterato dai perimetrali del Duomo moderno. Qui è stato possibile osservare un frammento architettonico con scanalature (fig. 15), allettato nel primo corso dell'alzato (quindi in una posizione non troppo visibile), sopra il quale si osservava un filare parzialmente realizzato con alcuni pezzi litici di forma quadrangolare non decorati, uno dei quali di notevoli dimensioni. A parte quest'ultimo esemplare, messo in opera forse perché utile componente strutturale per la sua mole e ben visibile nel tessuto murario composto principalmente da laterizi, sembra che nemmeno in queste murature vi sia stata una selezione nella scelta degli spolia da utilizzare e, soprattutto, una volontà di esibirli. Un atteggiamento molto simile si è verificato anche in epoca successiva. Quando, nel corso del XVI secolo, si restaurò perimetrale di una cappella laterale, un grande capitello con il decorazione fitomorfa (uno dei pochi spolia che ha continuato a circolare nelle diverse fabbriche di età medievale) sembra essere stato utilizzato come mero materiale costruttivo: la faccia visibile all'interno della muratura è scalpellata e resa piana (fig. 15).

\section{GLI SPOLIA "ROVINATI": INDICE DI UNA CHIESA "DISTRUTTA"?}

Un interessante interrogativo è quello relativo alle cause e all'entità delle distruzioni della chiesa altomedievale alla quale dovevano appartenere gli spolia.
È certo che, qualsiasi fosse stato il degrado della chiesa primitiva, la fabbrica di età romanica comportò un totale riassetto topografico dell'area, precedentemente segnata dalla chiesa e dal cimitero. In quell'occasione avvenne il prelievo degli elementi costruttivi dell'aula originaria, cioè sia di quei manufatti che erano stati già reimpieghi nel secolo VIII, sia di quelli che, invece, avevano costituito il nuovo arredo liturgico intorno agli inizi del secolo IX. Se al nuovo impianto abbia corrisposto anche una precisa volontà distruttiva nei confronti della chiesa originaria, volta cioè a soddisfare una riqualificazione del complesso per diverse esigenze di spazi o per un mutamento del gusto, non siamo in grado di dirlo. Ci chiediamo, però, se il rinvenimento di ampi frammenti di colonne, utilizzati come brutale materiale costruttivo, non sia indizio del fatto che la struttura fosse crollata (almeno parzialmente), rendendo inservibili quelle componenti architettoniche che ci aspetteremmo essere preziosi elementi di recupero. A fronte della ricercatezza di cui furono oggetto, in questo periodo, i "marmi antichi", con modalità di utilizzo che contemplano anche la loro riduzione a piccole dimensioni-si pensi al serpentino e ad altri "verdi" impiegati nell'ambito delle arti orafe -, il caso di Comacchio pare di segno completamente opposto.

Purtroppo, limpossibilità di scavare le strutture originarie della chiesa episcopale, ci impedisce al momento di determinare se l'eventuale crollo sia stato l'esito di un incidente o, invece, di un'azione mirata. Le tracce di focatura, rilevabili su 
alcuni pezzi del recinto presbiteriale alto-medievale, tuttavia, potrebbero testimoniare l'evento di un incendio. In questo caso, si potrebbe tentare un'associazione, non poi troppo ardita, tra questo dato archeologico e quanto riportato dalle fonti scritte. Le cronache veneziane raccontano infatti dello sforzo intrapreso dalla flotta dogale contro il principale antagonista nel controllo e nella gestione dei traffici lagunari/ adriatici e mediterranei, cioè Comacchio. Nel 932, quindi nel primo trentennio del X secolo, il doge Pietro II Candiano avrebbe inviato un esercito contro i Comacchiesi, sferrando un attacco sull'abitato e sugli abitanti, che Giovanni Diacono definisce "terribile" (Giovanni diacono, Istoria Veneticorum, III, 44). Sebbene l'impresa possa essere stata enfatizzata, è impossibile non soffermarsi sulla coincidenza cronologica con quanto documentato dallo scavo, ossia la chiusura dellisola episcopale (centro nevralgico del potere) con terrapieno e fossato e la necessità di ricostruire la chiesa nel corso del secolo $\mathrm{XI}^{19}$.

Per quanto affascinante, questa non può restare che un'ipotesi, anche perché, stanti le focature, queste potrebbero anche essere la conseguenza di una successiva esposizione al fuoco, quella magari di una vicina calcara, che possiamo immaginare presente nel cantiere edilizio romanico.

Per riassumere, il complesso episcopale di Comacchio (chiesa ed edifici annessi) documenta diversi momenti di riuso degli spolia nel corso della sua lunga storia. Un primo riuso avvenne nella fase della primitiva costruzione, con l'arrivo di marmi, anche pregiati, forse dalla vicina Ravenna.
E' molto probabile che questi primi spolia riguardassero parti anche considerevoli dell'arredo (dalle lastrine parietali alle colonne e capitelli, forse per la divisione in navate della chiesa). In questo caso, i riusi sembrano ovviamente funzionali, ma legati a parti pregevoli e a vista della chiesa, dunque svolgevano la duplice funzione di reggere porzioni architettoniche dell'edificio, ma anche di dichiarare la ricchezza del suo arredo liturgico. I successivi riusi, invece, a partire da quello di epoca romanica, per finire con gli episodi ancora più tardi, sono tutti contrassegnati da un'identica cifra: la volontà di recuperare questi oggetti come materiale edile (al pari del pezzame laterizio). Il loro posizionamento nelle fondazioni dell'edificio, e non nell'alzato, rinforza questa spiegazione utilitaristica. Ciò potrebbe anche apparire strano, in un momento (l'epoca romanica) in cui altrove non è infrequente rilevare un uso pienamente ideologico degli spolia. Tuttavia, non solo dobbiamo riconoscere che il marmo antico esibito non è la regola neppure in questo periodo e in questa zona (per restare al territorio ferrarese, basti pensare al portale della chiesa di San Giorgio di Argenta, nel quale alcune stele funerarie romane furono usate per le nuove sculture romaniche), ma dobbiamo anche mettere nel conto le condizioni di conservazione in cui versavano questi oggetti al momento del loro reimpiego. Se davvero anche la chiesa episcopale di Comacchio, con l'abitato, dovette subire danni pesanti a seguito delle incursioni dei Venetici agli inizi del secolo $\mathrm{X}$, forse non è inspiegabile né irragionevole la decisione di farne del semplice materiale da costruzione.

* Dipartimento di Studi Umanistici, Università Ca' Foscari, Dorsoduro 3484/d - Venezia (30123), Italia

${ }^{1}$ S. GELICHI, Comacchio e il suo territorio tra la Tarda Antichità e l'Alto Medioevo, in Genti nel Delta da Spina a Comacchio. Uomini, territorio e culto dall'antichità all'altomedioevo, F. BERTI, M. BOLLINI, J. ORTALLI, S. GELICHI (a cura di), Ferrara, 2007, pp. 363-689; Idem, The eels of Venice. The long eight century of the emporia of the northern region along the Adriatic coast, in 774. Ipotesi su una transizione, S. GASPARRI (a cura di), Turnhout, pp. 81-117; Idem, Venice, Comacchio and the Adriatic Emporia between the Lombard and Carolingian ages, in Dorestad in an International Framework. New Research on Centres of Trade and Coinage in Carolingian Times, A. WILLEMSEN, H. KIK (eds.), Turnhout, 2010, pp. 149-157.

${ }^{2}$ S. GELICHI, D. CALAON, E. GRANDI, C. NEGRELLI, “... castrum igne combussit...”. Comacchio tra la Tarda Antichità e l'Alto Medioevo, in Archeologia Medievale XXXIII, Firenze, 2006, pp. 19-48; S. GELICHI, D. CALAON, E. GRANDI, C. NEGRELLI, Il quartiere episcopale di un emporio altomedievale. Gli scavi nel centro storico di Comacchio e la sequenza dei materiali, in L'archeologia dei paesaggi costieri e le variazioni climatiche. Il Progetto Interreg Italia Slovenia Alto Adriatico, R. AURIEMMA, S. KARINJA (a cura di), Trieste, 2009, pp.162-170.

${ }^{3}$ S. GELICHI, D. CALAON, E. GRANDI, S. LORA, C. NEGRELLI, Uno scavo scomposto. Un accesso alla storia di Comacchio attraverso le indagini presso la Cattedrale, in Missioni Archeologiche e progetti di ricerca e scavo dell'Università Ca' Foscari - Venezia, VI Giornata di Studio, Venezia, 12 maggio 2008, S. GELICHI (a cura di), Venezia, 2008, pp. 167-178; S. GELICHI, D. CALAON, E. GRANDI, C. NEGRELLI, II quartiere episcopale...

${ }^{4}$ L'Tsola del Vescovo. Gli Scavi Archeologici intorno alla Cattedrale di Comacchio - The Archaeological Excavations nearby the Comacchio Cathedral, S. GELICHI (a cura di), Firenze, 2009.

${ }^{5}$ S. GELICHI, D. CALAON, E. GRANDI, C. NEGRELLI, The History of a Forgotten Town: Comacchio and the Archaeology, in From one sea to another Trade centres in the Adriatic and Mediterranean in the Early Middle Ages, Proceedings of the International Conference in Comacchio (FE-Italy), March, 2009, S. GELICHI, R. HODGES (eds.), in print.

${ }^{6}$ S. GELICHI, D. CALAON, E. GRANDI, S. LORA, C. NEGRELLI, Uno scavo scomposto... ; S. GELICHI (a cura di), L'Isola del Vescovo..., pp. $44-46$.

${ }^{7}$ Sul cipollino, L. LAZZARINI, Poikiloi lithoi, versiculores macvulae: I marmi colorati della Grecia antica. Storia, uso, diffusione, cave, geologia, caratterizzazione scientifica, archeometria, deterioramento, in Marmora 2/Supplemento, Pisa-Roma, 2007, pp. 111-117 e bibliografia; sul verde antico (marmor thessalicum o lapis atracium); Ibidem, pp. 223-244 e bibliografia.

8 Sul giallo antico, E. DOLCI, L. NISTA, Marmi antichi da collezione. La Raccolta Grassi del Museo Nazionale Romano, Carrara, 1992, p. 67; sul rosso antico, L. LAZZARINI, I marmi colorati della Grecia antica..., pp. 71-96 e bibliografia.

9 Per pavimenti a sectilia di V e VI secolo e l'esempio della basilica placidiana di S. Croce a Ravenna, ove ricorrono analoghi elementi esagonali, F. GUIDOBALDI, Sectilia pavimenta e incrustationes: i rivestimenti pavimentali e parietali, in Eternità e nobiltà di materia. Itinerario artistico fra le pietre policrome, A. GIUSTI (a cura di), Firenze, 2003, pp. 54-55 e fig. 62.

${ }^{10}$ Tradizionalmente ritenuti pertinenti alla chiesa di S. Andrea dei Goti, demolita dai Veneziani nel 1453, R. FARIOLI, "Corpus” della scultura paleocristiana bizantina ed altomedioevale di Ravenna, III. La scultura architettonica: basi, capitelli, pietre d'imposta, pilastri, pilastrini, plutei, pulvini, Roma, 1969, n. 40, p. 31. Sui capitelli, che in alcuni casi presentano il monogramma di Teodorico, vedi anche P. NOVARA, La nascita del culto di Sant'Andrea a Ravenna e la Basilica di Sant'Andrea “dei Goti”, in Romagna arte e storia 6, 1986, pp. 10-11. La studiosa ricorda come i tre capitelli del Museo Nazionale 
siano giunti nelle collezioni museali da altre sedi, rispettivamente dal palazzo comunale, dalla pieve di Godo (dove uno di questi era riutilizzato come fonte battesimale), da una collezione privata. Per la questione della chiesa di S. Andrea, Ibidem, p.12.

${ }^{11}$ R. FARIOLI “Corpus" della scultura paleocristiana..., n. 41, pp. 31-32.

${ }^{12}$ Ibidem, p. 32. Un capitello teodoriciano di questa tipologia fu inoltre reimpiegato come acquasantiera nella pieve di Cesato, Ibidem, 40 , p. 31.

${ }^{13} \mathrm{Nel}$ primo frammento (fig. 12.a) è possibile leggere: --]eccati[--, interpretabile come [p]eccati; nel secondo frammento (fig. 12.b): --]ip[--. Le lettere dell'iscrizione presentano alcuni confronti con quelle incise negli elementi di arredo conservati presso il Museo Archeologico Nazionale di Ravenna (n. inv. 764). Per gli elementi ravennati, R. FARIOLI, “Corpus” della scultura paleocristiana bizantina ed altomedioevale di Ravenna, II. Altari, amboni, cibori, cornici, plutei con figure di animali, e con intrecci, transenne e frammenti vari, Roma, 1968, schede 32-33, p. 36.

${ }^{14}$ S. PATITUCCI UGGERI, Il sarcofago del vescovo-duca Stefano. Contributo alla storia di Comacchio nel secolo IX, in Analecta Pomposiana V, 1980, pp. 7-23; Eadem, Problemi storico-topografici di Comacchio tra tardo antico e alto medioevo: gli scavi di Valle Ponti, in Actes du XI Congrès International d'Archéologie Chrétienne (Lyon, Vienne, Grenoble, Genève et Aoste, 21-28 Septembre 1986), III, Roma, 1986, pp. 2301-2315; P. PORTA, Comacchio dalla Tarda Antichità all'Alto Medioevo: spunti per uno studio sui resti scultorei, in Genti nel Delta da Spina a Comacchio. Uomini, territorio e culto dall'antichità all'alto medioevo, Ferrara, 2007, pp. 473-484; Eadem, Evergetismo ecclesiastico tra medio e alto Adriatico: sculture altomedievali del territorio ferrarese, in Ideologia e cultura artistica tra Adriatico e Mediterraneo orientale (IV-X secolo): il ruolo dell'autorità ecclesiastica alla luce di nuovi scavi e ricerche, R. FARIOLI CAMPANATI et alii, (a cura di), Bologna, 2009, pp. 425-442.

${ }^{15}$ P. PORTA, Comacchio dalla Tarda Antichità all'Alto Medioevo..., p. 475, n. 4, frg. lastra con motivo ad intreccio da Valle Rabisola, ipoteticamente ricondotto alla chiesa cimeteriale di S. Mauro; nastro bisolcato con spigolo vivo in pilastrino o cornice, con n. 19, Ibidem, p. 480, da territorio di Comacchio non meglio specificato, ora Museo Archeologico di Ferrara.

${ }^{16}$ Un'eccezione ricorre forse in un caso, estremamente frammentario, edito in P. PORTA, Comacchio dalla Tarda Antichità all'Alto Medioevo..., p. 474, n. 1.

${ }^{17}$ Per esempio, è il caso dei capitelli frammentari con corona di archetti (fig. 12.c), confrontabili con gli esempi di Grado (lapidario), per cui A. TAGLIAFERRI, Corpus della scultura altomedievale, X. La diocesi di Aquileia e Grado, Centro Italiano di Studi sull'Alto Medioevo, Spoleto, 1981, p. 395, n. 615, tav. CCXXIV. ${ }^{18}$ Il catalogo degli elementi di arredo liturgico e dei restanti materiali lapidei rinvenuti durante le indagini archeologiche della cattedrale di Comacchio, curato da Riccardo Belcari, è in corso di pubblicazione nell'ambito dell'edizione dei risultati delle indagini archeologiche. Una presentazione preliminare in S. GELICHI (a cura di), L'Isola del Vescovo..., pp. 45-46; pp. 60-61.

${ }^{19}$ S. GELICHI, C. NEGRELLI, D. CALAON, E. GRANDI, Comacchio tra IV e X secolo: territorio, abitato e infrastrutture, in IV Congresso Nazionale di Archeologia Medievale (Scriptorium dell'Abbazia, Abbazia di San Galgano, Chiusdino, Siena, 26-30 Settembre, 2006), R. FRANCOVICH, M. VALENTI (a cura di), Firenze, 2006, pp. 114-123; S. GELICHI, D. CALAON, E. GRANDI, C. NEGRELLI, “... castrum igne combussit...”..., pp. 19-48.

\section{SPOLIJA U KONTEKSTU. REUPOTREBA U SREDNJOVJEKOVNOM EPISKOPALNOM KOMPLEKSU U COMACCHIU} SAŽETAK

Arheološka istraživanja koja su se provodila od 2006. do 2008. godine na Piazza XX Settembre u Comacchiu omogućila su da se detaljno istraže faze srednjovjekovnog episkopalnog kompleksa. Istraživani kontekst pokriva široki kronološki luk, od 6. stoljeća do novoga vijeka.

Faze prvog episkopalnog kompleksa mogu se datirati oko polovice 8. stoljeća: radi se o indirektnim podacima (područje rezervirano za ukope i portik), s obzirom da se prvotna crkva zasigurno nalazila na mjestu današnje.

Istraživanjima su otkriveni brojni arhitektonski elementi i ulomci popločenja crkve iz 8 - 9. stoljeća, neki od njih reupotrebljeni u mlađoj građevini. Dekorativni aparat morao je koristiti spolirane elemente koji su potjecali sa starijih građevina, ne nužno lokalnih. U karolinško doba crkva dobiva oltarnu ogradu, od koje su pronađeni fragmenti pluteja, stupića, trabeacije i kapitela.
Veliku pravokutnu građevinu okrenutu prema trijemu crkve, na osnovu dimenzija, kvalitete materijala i topografskog odnosa, možemo interpretirati kao krilo biskupske palače iz doba romanike, a datiramo ju između kraja 10. i početka 12. stoljeća.

Zidovi ove palače skrivaju iznimno veliki broj spolija iz ranije crkve, koja je očito morala biti u nekom trenutku pregrađena.

Analiza ovih nalaza, informacije o materijalima iz cijelog konteksta iskopa (poput podnih i zidnih tesera, mramornih ploča ili reupotrebljene kamene građe) nude nam zanimljive zaključke o dekorativnom programu ranosrednjovjekovnog episkopalnog kompleksa u Comacchiu, ali i pružaju mogućnost analize načina i vrsta reupotrebe na gradilištu romaničkog doba.

Prevela: Josipa Lulić 
